\title{
Demographic profile of Acute Pancreatitis in Eastern India: A Single Centre Experience
}

\author{
Dibyendu Mukherjee ${ }^{1}$, Shantanu Bhakta ${ }^{2}$, Sandeep Lahiry ${ }^{3}$, Rajasree Sinha ${ }^{4}$ \\ ${ }^{1,2}$ Associate Professor, Department of General Medicine, KPC Medical College \& Hospital, Kolkata, ${ }^{3}$ Postgraduate \\ trainee, Department of Pharmacology, Institute of Postgraduate Medical Education \& Research, (IPGME\&R), Kolkata, \\ ${ }^{4}$ Postgraduate trainee, Department of Pediatrics, Medical College \& Hospital, Kolkata
}

Background: Little is known about the etiological risk factors of acute pancreatitis (AP) in Eastern India. Aims and Objective: The aim of this study is to assess current trends in the etiology of AP in Eastern India. Materials and Methods: A retrospective study with crosssectional design was undertaken. It was based on patient records admit between January 2014 and January 2017 with physician-assigned diagnoses of AP at KPC Medical College \& Hospital, Kolkata $(n=234)$. Multivariate analyses were done to identify risk factors, and distribution was compared on the basis gender. Groups were selected on the basis of a working diagnosis. A stratified comparison was done in 3 commonest etiology groups identified: 'alcohol', 'non-alcohol' and 'idiopathic'. Results: The commonest etiologies were attributed to alcohol $(29.4 \%)$, idiopathic cause $(20.5 \%)$, and obstructive cause $(14.5 \%)$. Prevalence of AP was higher with alcoholism in men $(37 \%$ vs. $10.8 \% ; p<00.1)$, however, idiopathic cause $(12.3 \%$ vs. $37.8 \%)$, duct obstruction (1.2\% vs. $43.2 \%)$, obesity $(6.1 \%$ vs. $24.3 \%)$, and hypertriglyceridemia $(6.1 \%$ vs. $14.8 \%)$ were higher among females $(p<0.001$ for all comparisons). Compared to non-alcoholics, patients with idiopathic AP were more likely to be overweight ( $p=0.019)$ and have T2 DM ( $p=0.021)$. Impact assessment of T2 DM status further revealed that the risk of AP was even greater with obesity (Odds Ratio [OR] 1.37; $95 \%$ Confidence Interval $[\mathrm{Cl}] 0.57-3.26 ; p=0.047$ ) and smoking (OR $1.72 ; 95 \% \mathrm{Cl} 1.0$ $-2.97 ; p=0.049)$. Eighteen cases $(7.7 \%)$ of 'severe' AP were identified, mostly due to: trauma in $6(2.5 \%)$, idiopathic in $6(2.5 \%)$, gallstones in $4(1.7 \%)$, and alcoholism in $2(0.8 \%)$. Conclusion: Alcohol intake is the predominant etiological risk factor for acute pancreatitis in Eastern India. Gender and type 2 diabetes mellitus are important contributory determinants.

Access this article online Website:

http://nepjol.info/index.php/AJMS DOI: 10.3126/ajms.v8i6.18282 E-ISSN: 2091-0576 P-ISSN: 2467-9100

Key words: Acute pancreatitis, Risk factors, Diabetes Mellitus, Retrospective study, Cross-sectional study

\section{BACKGROUND}

Acute Pancreatitis (AP) is an inflammatory process due to autodigestion of the gland by pancreatic digestive enzymes, leading to impairment of function or any morphologic changes. ${ }^{1}$ It can recurr intermittently, contributing to ongoing insult, referred to as 'Chronic Pancreatitis' (CP). ${ }^{2}$ Severe AP (SAP) develops in about $25 \%$ of patients with AP. The average mortality rate in SAP approaches $2-10 \%{ }^{3}$

The incidence of AP is much higher in USA, Finland, and Scotland (49.3, 46.6 and 41.9 per 100,000 populations, respectively). ${ }^{4}$ In 2009 , it led to approximately 275,000 hospitalizations per year in the US. ${ }^{5}$ Hospitalization rates due to AP are found to increase progressively with age. ${ }^{6}$ For people aged 35-75 years, the rates double for males and quadruple for females. ${ }^{7}$ Blacks are 3 times more risk of developing AP to whites. ${ }^{8}$ However, little is known about the reasons for such racial disparity.

Conditions that can predispose to development of AP include: alcoholism, gallstones, abdominal surgery, certain medication re-exposures (didanosine, asparaginase, azathioprine, valproic acid, pentavalentantimonials, 
pentamidine etc.), tobacco smoking, cystic fibrosis, Type 2 Diabetes Mellitus (T2 DM), family history of pancreatitis, hypercalcemia (due to hyperparathyroidism), hypertriglyceridemia, infection, abdominal injury and pancreatic cancer. ${ }^{9}$ There are regional differences observed in demographic distributions of AP. For e.g. 'AlcoholRelated Pancreatitis' $(A R P)$ is more common in the West, compared to Asian countries. ${ }^{10-12}$ Prevalence is increased approximately 4-fold among subjects with a history of alcoholism. ${ }^{12}$ Alcohol consumption has been steadily increasing in developing countries, such as China and India, due to rapid urbanization and increased affluence, there has been a gradual shift mimicking the western world trend. ${ }^{13} A R P$ is more common in men, though gender differences disappear with similar levels of alcohol consumption. ${ }^{7} \mathrm{AP}$ in women is more likely related to gallstones, procedures like endoscopic retrograde cholangiopancreatography (ERCP), autoimmune diseases, or idiopathic disease ${ }^{14}$ Common etiologies of $\mathrm{AP}$ in pediatric patients include gallstones, medication, and idiopathic cause. ${ }^{15}$ Advances in diagnostic imaging, molecular \& cell biology and genetics, have broadened the list of possible etiologies. The number of presumed 'idiopathic' cases (10$15 \%$ ) will decrease as our understanding of the disease improves. ${ }^{16}$ However, the etiology should be determined as early as possible to allow physicians to choose appropriate treatment strategies.

Since, little is known about the etiological risk factors of AP in Eastern India, the specific aim of this study was to assess current trends etiology of AP in Eastern India.

\section{MATERIALS AND METHODS}

\section{Ethics}

The study was conducted in accordance with the Helsinki II declaration and protocol was approved by the Institutional Ethics Committee (IEC) at KPC Medical College \& Hospital, Kolkata, India.

\section{Study design}

A retrospective study with cross sectional design was undertaken based on available medical records of patients admit between January 2014 and January 2017 with physician-assigned diagnoses of AP at KPC Medical College \& Hospital, Kolkata $(n=234)$. Patients admit with a physician-assigned diagnosis of AP were enrolled and followed up. Data relating to clinical phenotype, risk factors, diagnostic and therapeutic interventions were noted and analyzed subsequently. Disease severity was assessed by Acute Physiology and Chronic Health Evaluation (APACHE) ${ }^{17}$ scoring system and available contrast-enhanced computed tomography scan reports (if any).
Multivariate analyses were done to identify risk factors, and distribution was compared on the basis gender. Groups were selected on the basis of a 'working diagnosis' (WD). A stratified comparison was done in 3 commonest etiology groups identified: 'alcohol', 'nonalcohol' and 'idiopathic'. All patients other than those belonging to 'alcohol' or 'idiopathic' etiology group were included in a single category of 'non-alcohol' group for comparison of demographics, risk factors, symptoms, and phenotypic characteristics. In addition, the prevalence of select 'risk modifiers' was identified: tobacco smoking, alcohol use, history of chronic kidney disease. Tobacco exposure was measured as 'packs per year' $[1$ pack $=20$ cigarettes $]$.

'At-risk' drinking, was identified from TWE $A K$ questionnaire $^{18,19}$ data, which is a validated measure, defined as a score of 3 or higher, taken during index visit. A composite score (range, 0-7) was calculated based on patient responses, and classified into one of 5 drinking categories: (i) abstainer (no alcohol use or $<20$ drinks in lifetime); (ii) light ( $\leq 0.5$ drinks/day); (iii) moderate ( $>0.1$ drink/day for females, $>0.5-2$ drinks/day for males); (iv) heavy ( $>1$ to $<5$ drinks/day for females, $>2$ or $<5$ drinks/ day for males), and (v) very heavy ( $\geq 5$ drinks/day for both genders). One 'standard' drink was defined as roughly 14 grams of pure alcohol, which is found in 12 ounces of regular beer ( $5 \%$ alcohol); 5 ounces of wine ( $12 \%$ alcohol); 1.5 ounces of distilled spirits ( $40 \%$ alcohol) ${ }^{20}$ Details of all documented adverse events (AE) during the study period were reviewed. Severity and causality were assessed using Modified Hartwig and Seigel scale and Naranjo's Algorithm, respectively. ${ }^{21,22}$

\section{Statistical analysis}

Descriptive analyses were presented as proportions for categorical data and as mean \pm standard deviation (SD) or median and interquartile range (IQR) for continuous data, as applicable. Continuous variables were compared using the Student $t$ test, and for categorical data using chisquare test, as applicable. Multivariate analysis was done to identify risk factors. The association between smoking, alcohol, and AP was assessed using multivariable logistic regression analyses. Alcohol consumption (as drinking categories), age (as a continuous variable), sex (male, female), prior history of CP or cholecystectomy, T2 DM, current or maximum BMI (normal/low $<25$; overweight: 25 to 29; obese $\geq 30$ ), and phenotypic features (exocrine insufficiency, endocrine insufficiency, duct dilation/ strictures and calcifications) were kept as covariates. A 2 -sided $P$ value $<0.05$ was defined as statistically significant. Analysis was performed using SPSS version 21 (SPSS, Inc, Cbicago, Illinois). 


\section{RESULTS}

In total, 234 adult patients were recruited. WD cited in most cases were similar for both genders $(p=0.42)$. The mean age of patients at the time of enrollment was $44.4 \pm 10.8$ years; $69.2 \%$ were males and $31.6 \%$ females. The commonest etiology was attributed to alcohol (29.4\%), idiopathic cause $(20.5 \%)$, and obstructive cause $(14.5 \%)$. Prevalence of AP was higher with alcoholism in men (37\% vs. $10.8 \%$; $p<00.1)$, however, other causes like idiopathic cause $(12.3 \%$ vs. $37.8 \%)$, duct obstruction $(1.2 \%$ vs. $43.2 \%)$, obesity (6.1\% vs. $24.3 \%)$, and hypertriglyceridemia (6.1\% vs. $14.8 \%)$ were higher among females $(p<0.001$ for all comparisons). Common obstructive causes included gallstones (20\%) and pancreas divisum (9.4\%). Other commonly encountered etiologies included: obesity $(11.9 \%)$, hypertriglyceridemia $(8.9 \%)$, and hereditary factors $(3.4 \%)$. Miscellaneous causes included trauma $(2.5 \%)$, hypercalcemia $(1.7 \%)$, radiation exposure $(0.8 \%)$, autoimmune conditions $(0.8 \%)$ and cystic fibrosis $(0.4 \%)$. (Table 1$)$

Trends were observed in subjects were distribution based on etiology groups. While more males were assigned to 'alcohol' (88.4\%) \& 'non-alcohol' group (69.2\%), the proportion of females in 'idiopathic' group was higher $(58.3 \%, p<0.05)$. A history of CP was present in majority of subjects $(62.3 \%)$. Chronic kidney disease was less frequently reported in the 'idiopathic' group (6.2\%). For most other variables, comparisons were similar between groups. Among patients where the physician had reported 'alcohol' as a WD, 69.5\% in 'alcohol' group were found to have self-reported at-risk drinking (based on TWEAK questionnaire taken during index visit). This was much higher compared to 'idiopathic' group $(10.4 \%)(p=0.050)$, after controlling for age, sex, BMI, tobacco smoking (OR
1.65; 95\% CI, 1.08 -2.52). High prevalence of tobacco smoking was found in the 'alcohol' group (78.2\%). The prevalence of obesity, defined as a maximum BMI of $\geq$ 30,were similar across all groups, however, patients in 'nonalcohol' group $(63.2 \%)$ were significantly more overweight, compared to 'alcohol' $(37.6 \%, p=0.032)$ and 'idiopathic' group $(45.8 \%, p=0.019)$. Phenotypic characteristics including exocrine and endocrine insufficiency and pancreatico-biliary duct abnormalities were comparable across groups. Such results provided important information on proximal etiologies that could be linked to pancreatic pathologies via unknown mechanism. (Table 2)

Overall, $65.3 \%$ patients had a history T2 DM on admission, and about $56.2 \%$ were smokers. However, the prevalence of obesity, dyslipidemia, alcohol addiction was relatively lower. Among comorbidities, obesity (odds ratio [OR] 1.37; 95\% confidence interval [95\% CI], $0.57-3.26 ; \mathrm{p}=0.047$ ) and smoking (OR 1.72; 95\% CI, 1.08-2.97; $\mathrm{p}=0.049)$ had a significant impact on the risk of AP in T2 DM. Among other co-morbidities, alcoholism, hypertriglyceridemia represented a strong impact on the risk of AP, but represented few cases. (Table 3)

Eighteen cases of severe acute pancreatitis were reported, mainly due to: trauma in $6(2.5 \%)$, idiopathic in $6(2.5 \%)$, gallstones in $4(1.7 \%)$, and alcoholism in $2(0.8 \%)$. However, there were no deaths reported, and all patients were treated according to a designed protocol. There were 12 episodes of adverse drug reactions (ADRS) reported, namely, hypoglycemia ( 9 ADRs of Moderate L3 and 3 ADRs of Moderate L4 (a)) in T2 DM patients receiving insulin (mostly pre-mixed insulin), which was averted by decreasing the daily dosage. 8 discrete episodes of gastrointestinal discomfort (6 ADRs of Mild L1 and 2 ADRs of Mild L2)

\begin{tabular}{|c|c|c|c|c|}
\hline Working diagnosis & Total $(n=234)$ & Female $(n=74)$ & Male $(n=162)$ & $p$ value \\
\hline Alcohol & $69(29.4)$ & $8(10.8)$ & $61(37.6)$ & $<0.001$ \\
\hline Idiopathic & $48(20.5)$ & $28(37.8)$ & $20(12.3)$ & $<0.001$ \\
\hline \multicolumn{5}{|l|}{ Non-alcohol } \\
\hline Obstructive & $34(14.5)$ & $32(43.2)$ & $2(1.2)$ & $<0.001$ \\
\hline Gall stones & $22(9.4)$ & $20(27)$ & $2(1.2)$ & \\
\hline Pancreatic divisum & $9(3.8)$ & $7(9.4)$ & $5(3)$ & \\
\hline Intraductal papillary neoplasm & $2(0.8)$ & $0(0)$ & $2(1.2)$ & \\
\hline Sphincter of Oddi dysfunction & $1(0.4)$ & $1(1.3)$ & $0(0)$ & \\
\hline Obesity & $28(11.9)$ & $18(24.3)$ & $10(6.1)$ & $<0.001$ \\
\hline Hypertriglyceridemia & $21(8.9)$ & $11(14.8)$ & $10(6.1)$ & \\
\hline Hereditary & $8(3.4)$ & $3(4)$ & $5(3)$ & \\
\hline \multicolumn{5}{|l|}{ Miscellaneous } \\
\hline Trauma & $6(2.5)$ & $1(1.3)$ & $5(3)$ & \\
\hline Hypercalcemia & $4(1.7)$ & $1(1.3)$ & $3(1.8)$ & \\
\hline Radiation & $2(0.8)$ & $0(0)$ & $2(1.2)$ & \\
\hline Auto-immune condition & $2(0.8)$ & $1(1.3)$ & $1(0.6)$ & \\
\hline Cystic fibrosis & $1(0.4)$ & $0(0)$ & $1(0.6)$ & \\
\hline Miscellaneous & $11(4.7)$ & $4(5.4)$ & $42(25.9)$ & \\
\hline
\end{tabular}

Data are presented as $n(\%)$ except where otherwise indicated. $P<0.05$ statistically significant 
Table 2: Demographics \& prevalence of risk factors in acute pancreatitis (stratified by Etiology groups)

\begin{tabular}{|c|c|c|c|c|c|c|}
\hline \multirow[t]{2}{*}{ Variable } & \multicolumn{3}{|c|}{ Etiology group } & \multicolumn{3}{|c|}{$p$ value } \\
\hline & $\begin{array}{c}\text { Alcohol } \\
(n=69)\end{array}$ & $\begin{array}{l}\text { Non alcohol } \\
\quad(n=117)\end{array}$ & $\begin{array}{l}\text { Idiopathic } \\
(n=48)\end{array}$ & $\begin{array}{l}\text { Alcohol vs } \\
\text { Non-alcohol }\end{array}$ & $\begin{array}{l}\text { Alcohol vs } \\
\text { Idiopathic }\end{array}$ & $\begin{array}{l}\text { Idiopathic vs } \\
\text { Non-alcohol }\end{array}$ \\
\hline \multicolumn{7}{|l|}{ Patient characteristics } \\
\hline Mean age $\pm S D$ & $49.8 \pm 11.6$ & $42.2 \pm 12.6$ & $41.3 \pm 8.4$ & 0.907 & 0.885 & 0.998 \\
\hline Males & $61(88.4)$ & $81(69.2)$ & $20(41.6)$ & 0.517 & 0.075 & $0.005^{*}$ \\
\hline Females & $8(11.5)$ & $38(32.4)$ & $28(58.3)$ & 0.236 & 0.517 & 0.845 \\
\hline History of chronic pancreatitis & $43(62.3)$ & $74(63.2)$ & $36(75)$ & 0.215 & 0.920 & 0.105 \\
\hline Prior cholecystectomy & $4(5.7)$ & $16(13.6)$ & $16(33.3)$ & 0.786 & 0.786 & $>0.999$ \\
\hline Type 2DM & $46(66.6)$ & $79(67.5)$ & $28(58.3)$ & 0.177 & 0.585 & $0.021^{*}$ \\
\hline \multicolumn{7}{|l|}{ Risk factors } \\
\hline 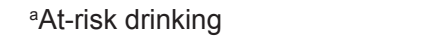 & $48(69.5)$ & $18(15.3)$ & $5(10.4)$ & 0.236 & $0.050^{*}$ & 0.754 \\
\hline Ever smoker & $54(78.2)$ & $56(47.8)$ & $26(54.1)$ & 0.993 & 0.282 & 0.236 \\
\hline \multicolumn{7}{|l|}{ Current BMI } \\
\hline Normal/low & $21(30.4)$ & $49(41.8)$ & $18(37.5)$ & 0.282 & 0.984 & 0.215 \\
\hline Overweight & $26(37.6)$ & $74(63.2)$ & $22(45.8)$ & $0.032^{*}$ & 0.097 & $0.019^{*}$ \\
\hline Chronic kidney disease & $18(26)$ & $26(22.2)$ & $3(6.2)$ & 0.891 & 0.687 & 0.420 \\
\hline \multicolumn{7}{|l|}{ Phenotypic features } \\
\hline Exocrine insufficiency & $11(15.9)$ & $32(27.3)$ & $9(18.7)$ & 0.484 & 0.993 & 0.420 \\
\hline Endocrine insufficiency & $13(18.8)$ & $36(30.7)$ & $6(12.5)$ & 0.420 & 0.920 & 0.236 \\
\hline Pancreatic duct dilation/strictures & $26(37.6)$ & $48(41)$ & $24(50)$ & 0.452 & 0.993 & 0.390 \\
\hline Calcifications & $28(40.5)$ & $45(38.4)$ & $6(12.5)$ & 0.619 & 0.452 & 0.084 \\
\hline CBD dilation/strictures & $9(13)$ & $4(3.4)$ & $3(6.2)$ & 0.958 & 0.941 & 0.998 \\
\hline
\end{tabular}

Data are presented as $n(\%)$ except where otherwise indicated. Current BMI was calculated based on the patient's weight at the time of enrollment. BMI: normal/low: $<25$; overweight: $25-29$; and obese $\geq 30$. BMI, body mass index [expressed as weight $(\mathrm{kg}) /$ height $\left(\mathrm{m}^{2}\right)$ ], alndividuals who met criteria for at-risk drinking (based on TWEAK questionnaire taken at index visit). $p$ value $<0.05$ statistically significant.

\begin{tabular}{|c|c|c|c|c|c|c|}
\hline Baseline co-morbidity & Non-diabetes $(n=81)$ & Type 2 diabetes $(n=153)$ & OR & $(95 \% \mathrm{Cl})$ & Z statistic & $p$ value \\
\hline \multicolumn{7}{|l|}{ Alcoholism } \\
\hline Yes & $23(28.3)$ & $46(30)$ & 1.08 & $0.59-1.96$ & 0.26 & 0.789 \\
\hline No & $58(71.6)$ & $107(69.9)$ & & & & \\
\hline \multicolumn{7}{|l|}{ Gall stones } \\
\hline Yes & $12(14.8)$ & $22(14.3)$ & 0.96 & $0.45-2.06$ & 0.09 & 0.928 \\
\hline No & $69(85.1)$ & $131(85.6)$ & & & & \\
\hline \multicolumn{7}{|l|}{ Hypertriglyceridemia } \\
\hline Yes & $9(11.1)$ & $12(7.8)$ & 0.68 & $0.27-1.69$ & 0.82 & 0.407 \\
\hline No & $72(88.8)$ & 141 (92.1) & & & & \\
\hline \multicolumn{7}{|l|}{ Obesity } \\
\hline Yes & $8(9.8)$ & $20(13)$ & 1.37 & $0.57-3.26$ & 0.71 & 0.047 \\
\hline No & $73(90.1)$ & $133(86.9)$ & & & & \\
\hline \multicolumn{7}{|l|}{ Smoking } \\
\hline Yes & $40(61.7)$ & $96(56.2)$ & 1.72 & $1.08-2.97$ & 1.96 & 0.049 \\
\hline No & $41(38.2)$ & $57(43.7)$ & & & & \\
\hline
\end{tabular}

Cl: Confidence interval; OR: Odds ratio.p value <0.05 statistically significant. OR: adjusted for age and mutually adjusted for co-morbid Hypertriglyceridemia, Alcoholism, Obesity \& Gallstones. BMI: normal/low: <25; overweight: 25-29; and obese $\geq 30$. BMI, body mass index [expressed as weight $(\mathrm{kg}) / \mathrm{height}\left(\mathrm{m}^{2}\right)$ ]

due to Metformin-induced AP (dose > 1gm/day), was recorded mostly in patients with chronic kidney disease. However, none of the ADRs were severe and had an uneventful course. Among all the ADRs ( $n=20), 6$ ADRs were of 'possible' category, and 2 were of 'probable' category on the causality assessment scale.

\section{DISCUSSION}

Our study observe interesting etiologic profile patterns in AP. Compared to a previous study at All India
Institute of Medical Sciences (AIIMS), New Delhi' ${ }^{23}$, we recorded a higher prevalence of AP in Eastern India (234 patients recorded in 36 months, average of 6 patients per month). Although gallstones have been implicated as a predominant cause of AP in many studies $^{24-27}$, majority cases in our study were alcoholrelated. However, in a large number of cases were associated with an'idiopathic'etiology. There are several potential explanations for such a trend. First, patients who are actively drinking in urban areas are more likely to seek a referral, than patients who are referred from 
rural centers. Second, most of our subjects were referred from primary or secondary health-care centers, which may be subject to referral bias. Tertiary medical centers are better equipped to handle conditions requiring surgical interventions, like gallstone disease; hence most patients are likely to seek a referral. Third, in most medical centers, diagnosis is mainly based on laboratory reports (hyperamylasemia), and access to a diagnostic imaging is limited, thus there is a delay in exclusion of causes like obstructive or anatomical abnormalities at an earlier stage. Fourth, changing lifestyle pattern over past 10 years has increased the risk of alcohol related pancreatitis in India.

We also recorded higher incidence of AP in T2 DM (approximately two-folds greater than that in the nondiabetic group). The risk of subsequent AP in T2 DM was relatively higher in the setting of obesity (1.37 fold) since obesity promotes gallstone formation ${ }^{28}$, and tobacco smoking (1.72 fold). However, the stratified risk estimates by co-morbidities may be imprecise because of limited number of cases. In addition, we did not compare the $\mathrm{HbA1c}$ level with the number of drugs used, a potential confounding situation, since few studies have shown a lower incidence of AP with lower HbA1c level due to metformin or glyburide use..$^{29,30}$

It is interesting to note that in $70.6 \%$ cases, alcohol was not found to be the predominant etiological risk factor. The diagnosis was based on patient evaluation at the time of enrollment. A diagnosis of an idiopathic cause reflected improvements in the extent of diagnostic evaluation. However, having a cross-sectional design, follow-up data was not analyzed for diagnostic trends, which could have changed in the natural history of the disease. Demographic and phenotypic characteristics in 'idiopathic' group were similar to other groups, except for prevalence of higher BMI (overweight). Interestingly, at-risk drinking or tobacco smoking in 'non-alcohol' group was comparable to 'alcohol' group. Univariate analysis also could not reveal a significant association between AP and other covariates like renal disease, gender predilection, or a history of $\mathrm{CP}$ or T2 DM.

Thus, our study could reveal important trends in the etiology of AP in Eastern India. It strength lied in a significantly large data set and assessment of impact of T2 DM on incidence of AP. However, there were several limitations too, some of which included (i) the study was unicentric, and therefore may not be representative in a wider context; (ii) the data used for this study came from a cohort of a managed care population;thus, results are applicable primarily to the prevalence of outcomes in managed care settings; (iii) a cross-sectional design, may have lent itself to retrospective bias; (iv) a longitudinal study design with regular follow-up could have analyzed for diagnostic trends; (v) physician working diagnosis are susceptible to the extent of diagnostic evaluation during enrollment and to individual physician biases; (vi) use of tests to measure serum amylase, which detect milder cases of AP could also result in over-diagnosis; (vii) physician interpretation of alcohol related pancreatitis may be variable; (viii) results were probably conservative, given that subjects with 'pre-diabetes' may have been included in the non-diabetic cohort.

\section{CONCLUSION}

Alcohol has become the predominant etiological risk factor for acute pancreatitis in Eastern India recently. Gender and type 2 diabetes mellitus are important determinants in etiology of acute pancreatitis in Eastern India. Future studies may explain environmental and genetic interactions modifying disease development.

\section{REFERENCES}

1. Klöppel G and Maillet B. Pathology of acute and chronic pancreatitis. Pancreas 1993;8:659-670.

2. Witt H, Apte MV, Keim V and Wilson JS. Chronic pancreatitis: challenges and advances in pathogenesis, genetics, diagnosis, and therapy. Gastroenterology 2007;132:1557-1563.

3. Surbatovic $M$ and Radakovic $S$. Tumor necrosis factor- $\alpha$ levels early in severe acute pancreatitis: is there predictive value regarding severity and outcome? J Clin Gastroenterol 2013;47:637-643.

4. Toouli J, Brooke-Smith M, Bassi C, Carr-Locke D, Telford J, Freeny $\mathrm{P}$, et al. Guidelines for the management of acute pancreatitis. J Gastroenterol Hepatol 2002;17 Suppl: S15-S39.

5. Peery AF, Dellon ES, Lund J, Crockett SD, McGowan CE, Bulsiewicz WJ, et al. Burden of gastrointestinal disease in the United States: 2012 update. Gastroenterology 2012;143:1179-1187.

6. Lowenfels $\mathrm{AB}$, Maisonneuve $\mathrm{P}$ and Sullivan $\mathrm{T}$. The changing character of acute pancreatitis: epidemiology, etiology, and prognosis. Curr Gastroenterol Rep 2009; 11:97-103.

7. Lankisch PG, Lowenfels $A B$ and Maisonneuve P. What is the risk of alcoholic pancreatitis in heavy drinkers? Pancreas 2002; 25:411-412.

8. A.L. Yang, Vadhavkar S, Singh G and Omary MB. Epidemiology of alcohol-related liver and pancreatic disease in the United States. Arch Intern Med 2008;168:649-656.

9. Yadav D and Lowenfels AB. Trends in the epidemiology of the first attack of acute pancreatitis: a systematic review. Pancreas 2006; 33:323-330.

10. Yadav $D$ and Whitcomb DC. The role of alcohol and smoking in pancreatitis. Nat Rev Gastroenterol Hepatol 2010; 7:131-145.

11. HN Shen, CL Lu and CY Li. Epidemiology of first-attack acute pancreatitis in Taiwan from 2000 through 2009: a nationwide population-based study. Pancreas 2012;41:696-702.

12. Ramstedt M. Alcohol and pancreatitis mortality at the population level: experiences from 14 western countries. Addiction 2004;99:1255-1261. 
13. Global Status Report on Alcohol and Health 2011 [online]. Available from: http://www.who.int/substance_abuse/publications/ global_alcohol_report/en/index.html. [ Accessed March 24, 2017]

14. Testoni PA. Acute recurrent pancreatitis: Etiopathogenesis, diagnosis and treatment. World J Gastroenterol 2014;20:16891-16901.

15. Bai HX, Lowe ME and Husain SZ. What have we learned about acute pancreatitis in children? J Pediatr Gastroenterol Nutr 2011;52: 262-270.

16. DiMagno MJ and DiMagno EP. New advances in acute pancreatitis. Curr Opin Gastroenterol 2007;23:494.

17. Niskanen M, Kari A, Nikki P, Kaukinen L, Rauhala V, Saarela E, et al. Acute physiology and chronic health evaluation (APACHE II) and Glasgow coma scores as predictors of outcome from intensive care after cardiac arrest. Crit Care Med1991;19:1465-1473.

18. Chan AW, Pristach EA, Welte JW and Russell M. Use of the TWEAK test in. Screening for alcoholism/heavy drinking in three populations. Alcohol Clin Exp Res 1993;17:1188-1192.

19. Yadav D, Hawes RH, Brand RE, Anderson MA, Money ME, Banks PA, et al. Alcohol consumption, cigarette smoking, and the risk of recurrent acute and chronic pancreatitis. Arch Intern Med 2009;169:1035-1045.

20. Dietary Guidelines for Americans 2015-2020. U.S. Department of Health and Human Services and U.S. Department of Agriculture [online]. Available from: https://health.gov/ dietaryguidelines/2015/guidelines/appendix-9/. [ Accessed March 24, 2017]
21. Hartwig SC, Siegel J and Schneider PJ. Preventability and severity assessment in reporting adverse drug reactions. Am J Hosp Pharm 1992;49:2229-2232.

22. Naranjo CA, Busto U, Sellers EM, Sandor $P$, Ruiz I and Roberts EA. Naranjo ADR probability scale. Clin Pharmacol Ther 1981;30:239-245.

23. Tandon RK. Management of Acute Pancreatitis: Indian Guidelines and Protocols. API Medicine Update 2013;23:267-270.

24. Dammann HG, Döpner M, Wichert PV, Harders $H$ and Hornborstel H. Gallstones and acute pancreatitis. The Lancet 1980;315:308.

25. Wettendorff P, Van Gossum A and Delcourt A. Gallstones and acute pancreatitis. The Lancet 1981;317:675.

26. Artigas JG, Gali FG, Siscart MP and Faure MA. Gallstones and acute pancreatitis. The Lancet 1981;317:947-948.

27. McMahon MJ and Pickford IR. Gallstones and acute pancreatitis. The Lancet 1980;315:545-546.

28. Biddinger SB, Haas JT, Yu BB, Jing E, Zhang W, Unterman TG, et al. Hepatic insulin resistance directly promotes formation of cholesterol gallstones. Nature medicine 2008;14:778-782.

29. Gonzalez-Perez A, Schlienger RG and García Rodríguez LA. Acute pancreatitis in association with type 2 diabetes and antidiabetic drugs: a population based cohort study. Diabetes Care 2010;33:2580-2585

30. Blomgren KB, Sundstrom A, Steineck $G$ and Wiholm BE. Obesity and treatment of diabetes with glyburide may both be risk factors for acute pancreatitis. Diabetes Care 2002;25:298-302.

\section{Authors Contribution:}

DM- Concept and design of the study, manuscript preparation, statistically analyzed and interpreted, critical revision of the manuscript; SB- Concept and design of the study, critical revision of manuscript and review of the study; SL- reviewed the literature, helped in preparing first draft of manuscript, collected data; RS- collected data, statistically analyzed and interpreted, helped in preparing first draft of manuscript.

\section{Orcid ID:}

Dr. Dibyendu Mukherjee: http://orcid.org/0000-0003-3264-2924

Dr. Shantanu Bhakta: http://orcid.org/0000-0001-7529-2894

Dr. Sandeep Lahiry: io http://orcid.org/0000-0002-6919-0530

Dr. Rajasree Sinha: http://orcid.org/0000-0002-8937-0459

Source of Support: Nil, Conflict of Interest: None declared. 